\title{
Two-dimensional spatiotemporal complexity in dual-delayed nonlinear feedback systems: Chimeras and dissipative solitons ${ }^{\circ}$
}

Cite as: Chaos 28, 103106 (2018); https://doi.org/10.1063/1.5043391

Submitted: 08 June 2018 . Accepted: 13 September 2018 . Published Online: 09 October 2018

D. Brunner (D) B. Penkovsky (D), R. Levchenko, E. Schöll, L. Larger, and Y. Maistrenko

COLLECTIONS

F This paper was selected as Featured
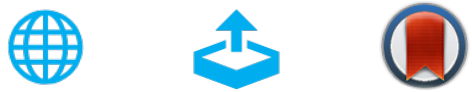

\section{ARTICLES YOU MAY BE INTERESTED IN}

Dissipative solitons for bistable delayed-feedback systems

Chaos: An Interdisciplinary Journal of Nonlinear Science 28, 101103 (2018); https:// doi.org/10.1063/1.5062268

Existence and stability of chimera states in a minimal system of phase oscillators Chaos: An Interdisciplinary Journal of Nonlinear Science 28, 103121 (2018); https:// doi.org/10.1063/1.5044750

Highly robust emergent 2-D patterns found in a time-delay laser system

Scilight 2018, 410003 (2018); https://doi.org/10.1063/1.5064595

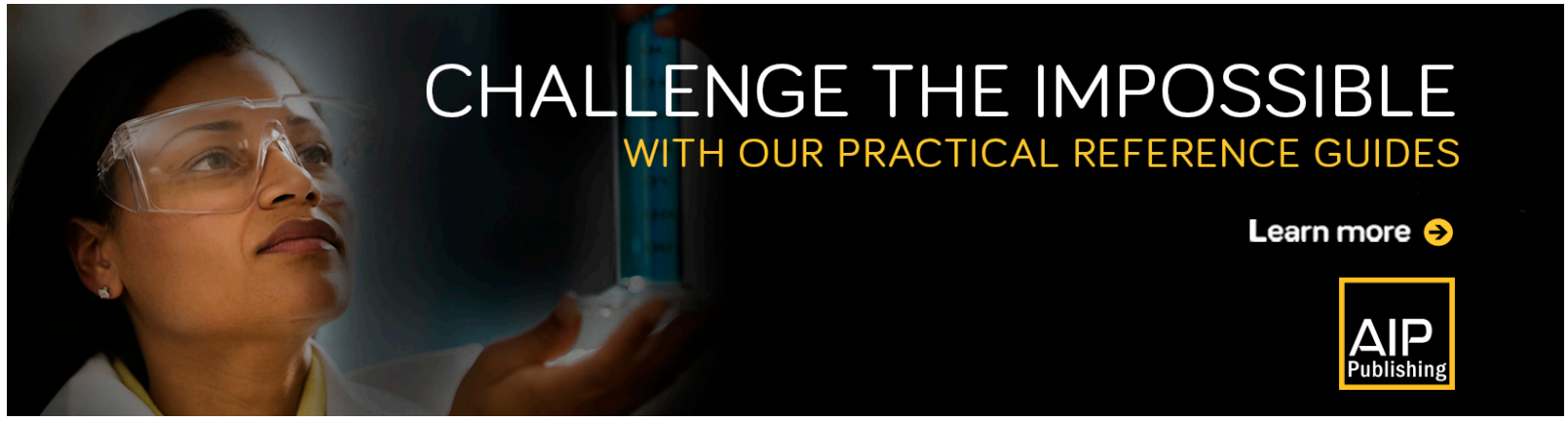




\title{
Two-dimensional spatiotemporal complexity in dual-delayed nonlinear feedback systems: Chimeras and dissipative solitons
}

\author{
D. Brunner, ${ }^{1}{ }^{1, a)}$ B. Penkovsky, ${ }^{1}$ R. Levchenko, ${ }^{2}$ E. Schöll, ${ }^{3}$ L. Larger, ${ }^{1}$ and Y. Maistrenko ${ }^{1,4}$ \\ ${ }^{1}$ FEMTO-ST Institute/Optics Department, CNRS \& University Bourgogne Franche-Comté, $15 B$ avenue des \\ Montboucons, 25030 Besançon Cedex, France \\ ${ }^{2}$ Taras Shevchenko National University of Kyiv, Volodymyrska St. 60, 01030 Kyiv, Ukraine \\ ${ }^{3}$ Institut für Theoretische Physik, Technische Universität Berlin, Hardenbergstraße 36, 10623 Berlin, Germany \\ ${ }^{4}$ Institute of Mathematics and Center for Medical and Biotechnical Research, NAS of Ukraine, Tereschenkivska \\ Str. 3, 01601 Kyiv, Ukraine
}

(Received 8 June 2018; accepted 13 September 2018; published online 9 October 2018)

\begin{abstract}
We demonstrate for a photonic nonlinear system that two highly asymmetric feedback delays can induce a variety of emergent patterns which are highly robust during the system's global evolution. Explicitly, two-dimensional chimeras and dissipative solitons become visible upon a space-time transformation. Switching between chimeras and dissipative solitons requires only adjusting two system parameters, demonstrating self-organization exclusively based on the system's dynamical properties. Experiments were performed using a tunable semiconductor laser's transmission through a Fabry-Pérot resonator resulting in an Airy function as nonlinearity. Resulting dynamics were bandpass filtered and propagated along two feedback paths whose time delays differ by two orders of magnitude. An excellent agreement between experimental results and the theoretical model given by modified Ikeda equations was achieved. Published by AIP Publishing. https://doi.org/10.1063/1. 5043391
\end{abstract}

Photonic delay systems are of astonishing diversity and have created a rich field of fundamental research and a wide range of applications. Under a transformation from time into pseudo-scape, their basic architecture makes them equivalent to ring networks with perfectlysymmetric coupling. For the first time we extend this spatiotemporal analogy in experiments by adding a second delay, 100 times the length of the first delay line. Nonlinearity is provided by a tunable semiconductor laser traversing a Fabry-Pérot resonator. Visualized in 2D-space, we show the temporal evolution of different chimeras and dissipative solitons. Experimental results excellently agree with numerical simulations of the double-delay bandpass Ikeda equation. Based on the attractors of multiple fixed-point solutions, we provide insight into the mechanism structuring the system's dynamics.

\section{INTRODUCTION}

The complex dynamical properties of high-dimensional nonlinear systems continue to create new and fascinating phenomena. Simple nonlinear equations or experimental systems are already capable of producing dynamics ranging from highly coherent motion all the way to hyper-chaos, in particular if they include time delays. ${ }^{1}$ Photonic systems provide paradigmatic examples, which have also great potential for applications, for instance, delay-coupled semiconductor lasers. ${ }^{2}$ Recently discovered chimera states ${ }^{3,4}$ are combinations of, both, chaotic and coherent motions within a symmetric network of identical elements, 5,6 they have

\footnotetext{
a) Electronic mail: daniel.brunner@femto-st.fr
}

been recently reviewed. ${ }^{7,8}$ This diversity stimulates not only continuous interest in the underlying principles, but it has also created a long-lasting output of novel applications. Nonlinear photonic systems have been identified as excellent substrates for highly coherent microwave oscillators, ${ }^{9}$ chaos encryption, ${ }^{10}$ neuromorphic processors, ${ }^{11,12}$ and regenerative photonic memory. ${ }^{13}$

The complex motion of nonlinear dynamical systems often reveal their underlying structure in the form of geometric patterns. These are readily found in the spatiotemporal dynamics of two dimensional (2D) substrates. Prominent examples are dynamics of Belousov-Zhabotinsky diffusion reactions, ${ }^{5}$ liquid crystal displays, ${ }^{14-16}$ or broad area semiconductor lasers. ${ }^{17-19}$ Yet, these phenomena are not limited to spatially extended systems, and comparable dynamical objects exist in nonlinear dynamical systems coupled to delay. ${ }^{1,20,21}$ Such nonlinear delay systems are heavily exploited in photonic technology, i.e., in mode-locked fiber lasers. Only recently, nonlinear delay dynamics have been found to sustain stable laser chimera $\operatorname{states}^{20}$ as well as dissipative solitons (DSs). ${ }^{21}$

Complex nonlinear dynamics found in delay systems relies on the finite propagation speed of the signal along the feedback path. In addition, the resulting propagation delay establishes a mapping between temporal and virtual space positions. ${ }^{22,23}$ After a normalization by approximately the delay-time, consecutive sections of unity length can be stacked. The results are dynamics in one continuous space dimension with a second dimension representing integer time. ${ }^{7,8,23,24}$ While spatially extended systems typically possess two dimensions, delay systems have so far been mostly limited to a single virtual space dimension. ${ }^{1}$ Here, we overcome this critical limitation and investigate pattern formation 
a

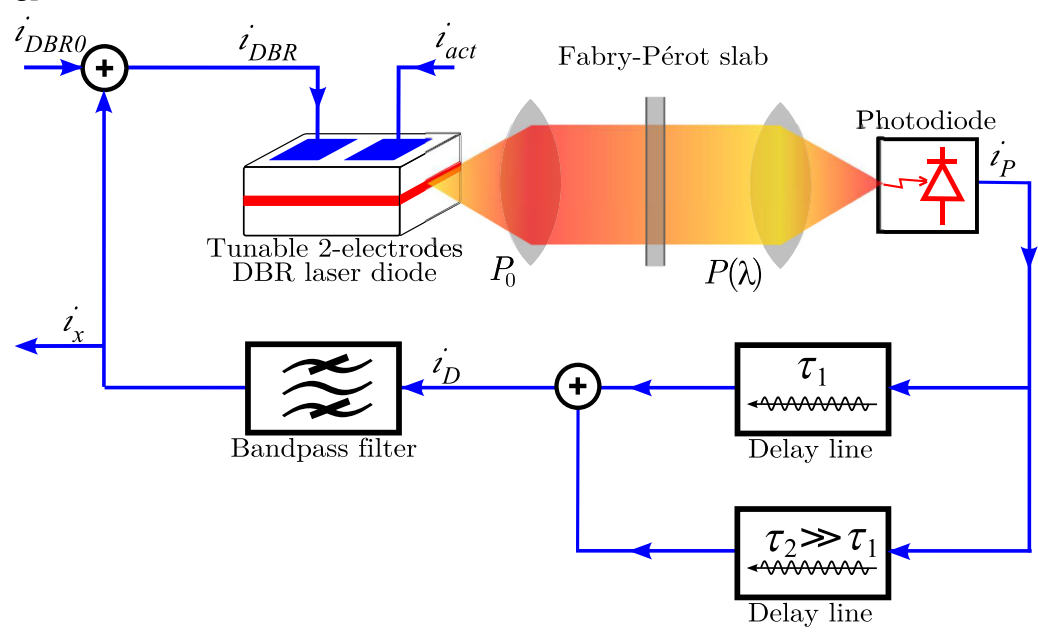

b
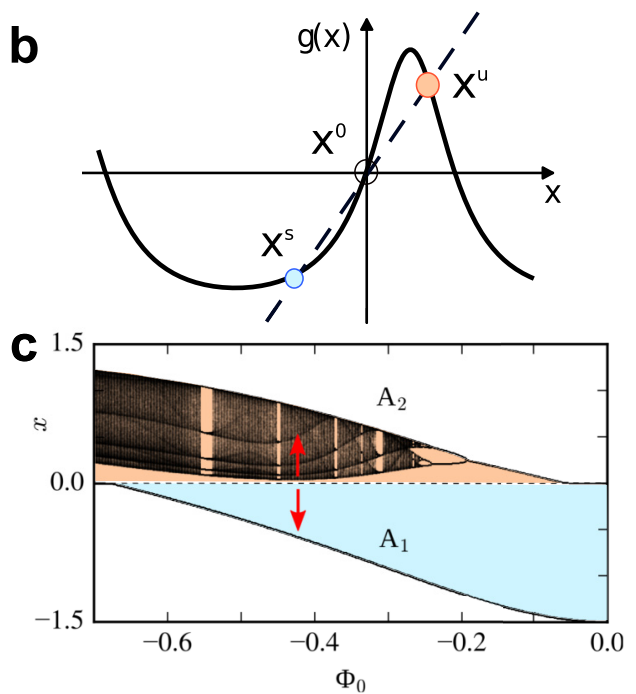

FIG. 1. Experimental setup and its nonlinear delay dynamics. (a) A semiconductor laser is subjected to two delayed optoelectronic feedbacks originating from a short $\tau_{1}$ and a long $\tau_{2} \gg \tau_{1}$ delay line. The delayed signals are bandpass filtered and injected into the DBR section of the laser diode, dynamically modulating its wavelength. (b) The optical emission is filtered by a Fabry-Pérot filter, creating an Airy function as the system's nonlinear response. In a one dimensional (1D) function $g(x)$, the asymmetry of the Airy function results in multiple fixed points $x^{s}, x^{u}$ and $x^{0}$, identified by its intersection with the shown first bisector (dashed line). (c) The 1D map's bifurcation diagram obtained via $x_{n+1}=g\left(x_{n}\right)$ with integer time $n$, with resulting dynamics concentrated around the system's fixed points. Red arrows indicate attracting direction for positive or negative initial conditions, identifying the range of dynamics found on attractor $\mathrm{A}_{1}$ (light blue) and $\mathrm{A}_{2}$ (light red). $\mathrm{A}_{1}$ corresponds to stable fixed point $x^{s}, \mathrm{~A}_{2}$ to an invariant attracting set born from the destabilization of initially stable fixed point $x^{u}$.

in a delay system coupled to two independent delays. A single photonic nonlinearity is coupled to feedback originating from two different feedback paths, where one delay exceeds the other by two orders of magnitude.

We report on multiple types of chimeras and DSs in a 2D virtual space consisting of a grid of coupled virtual photonic nonlinear oscillators. ${ }^{24}$ Assigning the evolution of the system's 2D space to a third dimension, chimeras and DSs form free standing three dimensional (3D) structures. Chimeras create columns consisting either of a coherent steady state or of chaotic dynamics, while the excitable nature of the DS manifests itself in spatial chaos ${ }^{25,26}$ due to a random spatial position. ${ }^{13,21}$ Extensive theoretical analysis accompanies our experimental investigations. Using delay differential equations (DDEs), we confirm our findings via an excellent agreement between numerical simulations and the experiment. Based on a reduced map equation, we identify the distribution of the dynamical variable during a particular state as the ordering mechanism of the different 2D dynamical objects, i.e., 2D chimeras and DSs.

\section{RESULTS}

In Fig. 1(a), we schematically illustrate the experimental setup. ${ }^{20}$ A tunable semiconductor single-mode laser diode, emitting at $\sim 1550 \mathrm{~nm}$, is biased at its gain section with a current of $i_{a c t}=20 \mathrm{~mA}$. The laser's wavelength is controllable via a second electrode, which supplies current $i_{D B R}$ to the distributed Bragg-reflector (DBR) section. ${ }^{24}$ As illustrated, the DBR electrode receives current from two sources $i_{D B R}=i_{D B R 0}+i_{x} . i_{D B R 0}$ is externally set to a constant value of around $10 \mathrm{~mA}$, fixing the central emission wavelength of the tunable laser close to the middle of a continuously tunable wavelength-range. The second contribution corresponds to the physical delay system's dynamical variable $i_{x}$, oscillating over a range of $\approx 4 \mathrm{~mA}$ leading to a continuous and linear tuning of the laser wavelength with negligible intensity modulations. The laser's optical intensity $P_{0}$ is detected after propagation through a Fabry-Pérot filter, making the detector's photo current $i_{P}(\lambda, t)$ a nonlinear function of the laser's wavelength at present. Our system's nonlinearity therefore corresponds to

$$
f(x)=\beta\left\{1+m \cdot \sin ^{2}\left[2 \pi\left(x+\Phi_{0}\right)\right]\right\}^{-1},
$$

where $\beta$ is a linear amplification. In our case, the Fabry-Pérot nonlinearity is created by transmission through two parallel reflective surfaces which create an optical filter due to optical interference; hence, $f(x)$ is the nonlinear Airy function. The spectral width of the filter's transmission is determined by $m$, which is a function of the resonator surfaces' reflectivity. Light back-reflected from the Fabry-Pérot or other components within the setup is filtered out by an optical isolator included on the laser-diode's package. The model's dynamical variable $x$ is unit-less and can be linked to the corresponding physical value $i_{x}$ via the laser's tuning-response $(\lambda / m A)$ and the Fabry-Pérot's sensitivity $(1 / \lambda)$. Physically, constant offset $\Phi_{0}$ is proportional to $i_{D B R 0}$, dynamical variable $x$ to $i_{x}$, and photocurrent $i_{P}$ to $f(x)$. More details on the system's wavelength-tuning mechanism and the Fabry-Pérot filter can be found in Ref. 24.

Photo-current $i_{P}$ is consecutively divided and delayed along two delay lines with signal delays $\tau_{1}=0.45 \mathrm{~ms}$ and $\tau_{2}=44.5 \mathrm{~ms}$. Both delays were implemented using first-in first-out memory blocks of a field programmable gate array (FPGA), and we chose $\tau_{2}=100 \times \tau_{1}$. The FPGA recombines both delayed signals according to $i_{D}(t)=(1-\gamma) i_{P}\left(t-\tau_{1}\right)+$ $\gamma i_{P}\left(t-\tau_{2}\right)$, where $\gamma$ allows for relative weighting between 
the two delays. Finally, $i_{D}$ is bandpass filtered and scaled with feedback gain $\beta$, creating current $i_{x}$. The bandpass filter is characterized by a low pass filter with a response time $\tau=12.7 \mu$ s cascaded with a high pass filter, the characteristic time of which is $\theta=160 \mathrm{~ms}$. The double delay loop is closed by injecting $i_{x}$ into the laser's DBR section.

In Fig. 1(b), we illustrate the nonlinear Airy function together with the first bisector. The first bisector corresponds to the diagonal line in the graph of the nonlinear function $g(x)$. In order to better understand the fixed points' properties, we evaluate the system's temporal evolution in discrete time $n$, based on a temporally-discrete mapping according to $x_{n+1}=g\left(x_{n}\right)$. Here, we use $g(x)=f(x)-f(0)$ and investigate the impact of constant phase-offset $\Phi_{0}$. The intersection of the first-bisector with $g(x)$ allows one to identify the map's fixed points. ${ }^{20}$ Multiples of those exist: one can be found on a positive slope located in a flat plateau, another on a steep negative slope close to the transmission maximum. In Fig. 1(c), we show the resulting bifurcation diagram for $m=4.7$ and $\beta=1.6$, which clearly demonstrates the impact which the asymmetry of $f(x)$ exerts upon the existence and stability of these fixed points. The small positive slope around $x^{s}$ results in the regular fixed-point attractor $\mathrm{A}_{1}$; the steep negative slope around $x^{u}$ gives rise to a chaotic attractor $\mathrm{A}_{2}$ via a perioddoubling cascade as parameter $\beta$ or $\Phi_{0}$ is varied to change the dynamical solution. Dynamics around these fixed points create basins of attraction, which are separated by the unstable fixed point $x^{0}$. Within both basins, the system's dynamical variable covers ranges $U\left[A_{1}\left(\Phi_{0}\right)\right]$ and $U\left[A_{2}\left(\Phi_{0}\right)\right]$, indicated via blue and orange areas in Fig. 1(c), respectively. Parameter $\Phi_{0}$ shifts function $g(x)$ along the horizontal axis and as such adjusts the ratio between $U\left(\mathrm{~A}_{1}\right)$ and $U\left(\mathrm{~A}_{2}\right)$. Combined with the bandpass filter's integrating effect, this ratio imposes a global structure upon the dynamics.

To better reveal the underlying pattern of the system's nonlinear dynamics, we translate the purely-temporal aspect's of the previous discussion into a $2 \mathrm{D}$ spatiotemporal frame. The objective of illustrating the system's dynamics in $2 \mathrm{D}$ pseudo-space is to clearly reveal symmetries consequence of the double delayed feedback architecture. As usual in bandwidth-limited systems, fundamental causalityconsiderations demand that reaction follows perturbation after certain temporal delay. A consequence of the system's delay architecture is that perturbations are the system's own temporally delayed state (ignoring noise). Dynamics should therefore reveal structures on the scale of the delays plus a causality-induced shift. We therefore introduce

$$
\begin{gathered}
\tilde{t}_{1}(t)=\frac{t}{\tau_{1}+\rho_{1}}, \\
\sigma_{1}(t)=\tilde{t}_{1}-\left\lfloor\tilde{t}_{1}\right\rfloor,
\end{gathered}
$$

as temporal normalization treating the short delay. Small constant $\rho_{1}$ is of the order of the bandpass filter's group delay, thus approximately the low-pass filter's characteristic time $\tau \ll \tau_{1}$; see Ref. 27. $\left\lfloor\tilde{t}_{1}\right\rfloor$ is the floor of $\tilde{t}_{1}$, i.e., the greatest integer that is less than or equal to $\tilde{t}_{1}$. Normalization according to Eq. (2) therefore focuses on effects on timescales $\sim \tau_{1}$, and $\sigma_{1}(t)$ is the position of $t$ within the normalization window.
The continuous time trace is then sectioned into temporally non-overlapping segments of length $\tilde{t}_{1}=1$. Concatenating these vectors creates a representation along $\sigma_{1}$ as the 1D pseudo-space, where the second dimension is integer time.

The impact of the long second delay now further structures the temporal evolution of the 1D pseudo-space, enabling its transformation into the second pseudo-space dimension according to

$$
\begin{gathered}
\tilde{t}_{2}(t)=\left\lfloor\tilde{t}_{1}\right\rfloor \frac{\tau_{1}+\rho_{1}}{\tau_{2}+\rho_{2}}, \\
\sigma_{2}(t)=\tilde{t}_{2}-\left\lfloor\tilde{t}_{2}\right\rfloor, \\
K(t)=\left\lfloor\tilde{t}_{2}\right\rfloor,
\end{gathered}
$$

where, as before, $\rho_{2}$ is a small constant related to the lowpass filter. The floor operator in Eq. (4) obtains the integer time resulting from the 1D pseudo-space mapping by Eqs. (2) and (3). Further scaling by the ratio between short and long delay echos relates dynamical features to the long delay line. Equation (5) finally maps scaled time $\tilde{t}_{2}(t)$ to the second pseudo-space dimension $\sigma_{2}(t)$ in analogy of the first time/pseudo-space transformation. As time $t$ progresses, complete frames in the $\left(\sigma_{1}, \sigma_{2}\right)$-plane are filled; $K(t)$ of Eq. (6) is the system's integer time for its evolution in complete $2 \mathrm{D}$ pseudo-space frames. ${ }^{28} \mathrm{~A}$ consequence of the floor operations in the introduced mappings is that pseudo-space dimension $\sigma_{1}$ is continuous, $\sigma_{2}$ is discrete, and $\sigma_{1}, \sigma_{2} \in[0,1]$.

\section{A. 2D laser chimeras}

Figures 2(a) and 2(b) show the temporal evolution of two different types of chimeras found in our experiment. Parameters were $\beta \approx 1.4, \Phi_{0} \approx-0.6$, and $m=33.5$. In Fig. 2(a), complex dynamics are broken into sections spaced by a steady state lasting $\sim \tau_{2}$. As revealed by the second, shorter time trace, dynamical motion inside a $\sim \tau_{2}$ window is further structured on temporal scales close to the short delay $\tau_{1}$. Generally speaking, the system predominantly resides in a steady state of the 1D-map model, regularly alternating with chaotic dynamics. The stable fixed point dynamics are located on attractor $A_{1}$, while chaotic motion resides on the chaotic attractor $A_{2}$. At the chosen $\Phi_{0}$, the stable fixed point's amplitude is less than the amplitude range covered by dynamics along the chaotic attractor. As a consequence, the majority of the time the system resides close to its stable fixed point, from where it makes excursions through the chaotic attractor's complex phase space. Globally, the pattern formed by these alternations is iteratively stable after the recurrence of the long delay.

In order to reveal the globally stable character, the full dynamical motion can be better captured after transformation into the spatiotemporal dimensions according to Eqs. (3), (5) and (6), with the resulting 3D representation shown in Fig. 2(c). Inside the $\left(\sigma_{1}, \sigma_{2}\right)$-plane, a region of chaotic dynamics is enclosed by the stable fixed-point state. Along the third dimension, we only show the boundary separating chaotic and stable motion. Showing the evolution of this state along the vertical discrete time dimension, and besides slight 

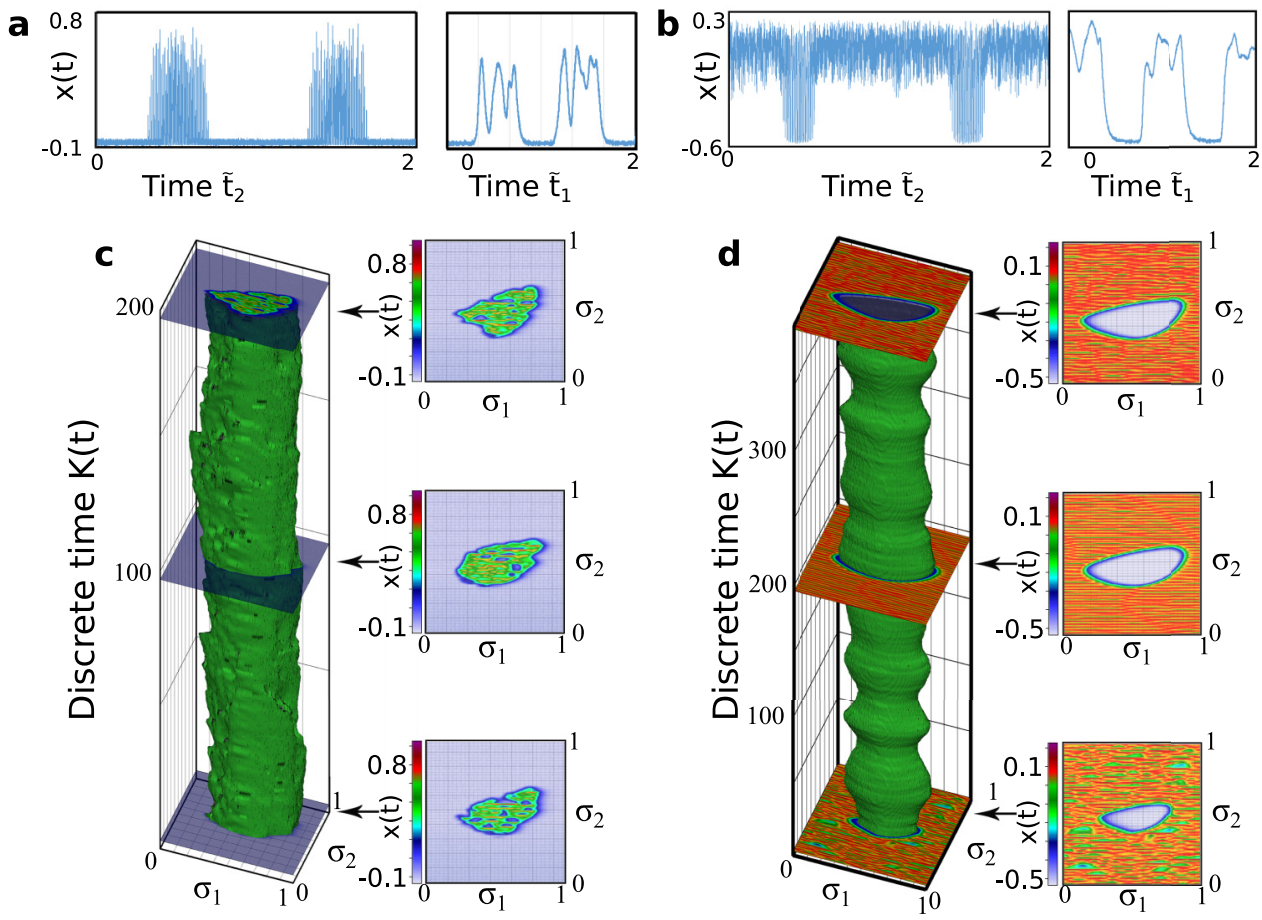

FIG. 2. Experimental laser chimeras. Asymptotic temporal waveforms of the chimeras during a long interval of the length $2 \tau_{2}$ and a zoom focusing on the short delay interval of the length $2 \tau_{1}$ are shown in panels (a) and (b). Corresponding space-time dynamics and respective snapshots of the chimeras in their $2 \mathrm{D}$-virtual space are shown in (c) and (d). Temporal evolution along the vertical dimension is accompanied by slow, chaotic breathing. Parameters are $\beta \approx 1.4$, $\Phi_{0}=-0.6, \gamma=0.5, m=33.5$ and $\beta \approx 0.7, \Phi_{0}=-0.05, \gamma=0.5, m=33.5$ for panels (a) and (c) and panels (b) and (d), respectively.

modifications, we demonstrate the long-term persistence of this 2D structure. As shown in Refs. 1 and 24, our 2D spatiotemporal system representation can be interpreted as a $2 \mathrm{D}$ network of nonlinear oscillators, each oscillator experiencing identical coupling and node parameters. Employing the analogy to such a network of Kuramoto phase oscillators, oscillators in the stable fixed point all share a common phase, while in the chaotic state no such uniform phase relationship exists. In our dual delay system, such symmetry breaking can exclusively be attributed to dynamical properties; the deviations from a perfect coupling symmetry, which can hardly be avoided in a $2 \mathrm{D}$ substrate, can here be excluded. Combined with the temporal stability, this identifies our dynamical state as the first demonstration of a chimera state along two virtual space dimensions of a double delay system.

Upon changing parameters to $\beta \approx 0.7, \Phi_{0} \approx-0.05$, the stable fixed point dynamics become surrounded by a sea of chaotic motion, as can be seen from data shown in Fig. 2(b). Similar to a harbor without physical walls inside a turbulent sea, this island of tranquility stably exists for longer than $350 \tau_{2}$. We therefore find that parameter $\Phi_{0}$ is an essential characteristic for the pattern formation in our system. Laser chimeras with incoherent core arise in the case when $U\left(A_{2}\right)$ is larger, while a larger $U\left(A_{1}\right)$ gives rise to the chimeras with a coherent core. Finally, for equal sizes of $U\left(A_{1}\right)$ and $U\left(A_{2}\right)$, we expect stripe-like chimera states. Both reported chimera states were obtained for $\gamma=0.5$, for which the short and long delayed feedbacks have equal amplitude weights. We generally find that chimera states arise under such balanced amplitude scaling conditions for both delays.

\section{B. Dissipative solitons}

Upon further exploration of the system parameters, we find that the chaotic attractor $\mathrm{A}_{2}$ can play a different, quite counter-intuitive role. Changing the offset-phase to more negative values, the attraction-basin $A_{2}$ continuously shrinks. As a result, $A_{2}$ loses its attracting property and is transformed into an unstable Cantor set. The states available to our system are therefore limited to the stable fixed point $x^{0}$ and a number of solutions arising under the influence of the attractor located in $U\left(\mathrm{~A}_{2}\right)$. Two characteristic examples of resulting dynamical states are illustrated in Figs. 3(a) and 3(b). Stable fixed point dynamics occupy the majority of the system's temporal evolution, interrupted by isolated spike-like structures. As we chose $\gamma=0.75$, the long delay's impact significantly dominates over the short delay. Consequently, dynamics are strongly regular on the $\tau_{2}$-scale but not on scale $\sim \tau_{1}$. We find that dynamics can experience one spike [Fig. 3(a)] or multiple spikes per delay $\tau_{2}$ [Fig. 3(b)].

Again, the global dynamical property can be better appreciated after the 2D transformation. The single spike of Fig. 3(a) corresponds to a single dissipative soliton (DS). ${ }^{21}$ The temporal evolution along the discrete long-time axis [Fig. 3(c)] reveals that this particular DS is born from chimera-like initial conditions which, in the course of time, transform into the asymptotic waveform of the DS. Data experiencing multiple spikes per $\sigma_{2}$ correspond to multiple DS structures; an example is shown in Fig. 3(d). Each of the shown DS structure was obtained by resetting the setup (blocking the laser beam), and multiple initializations of the 

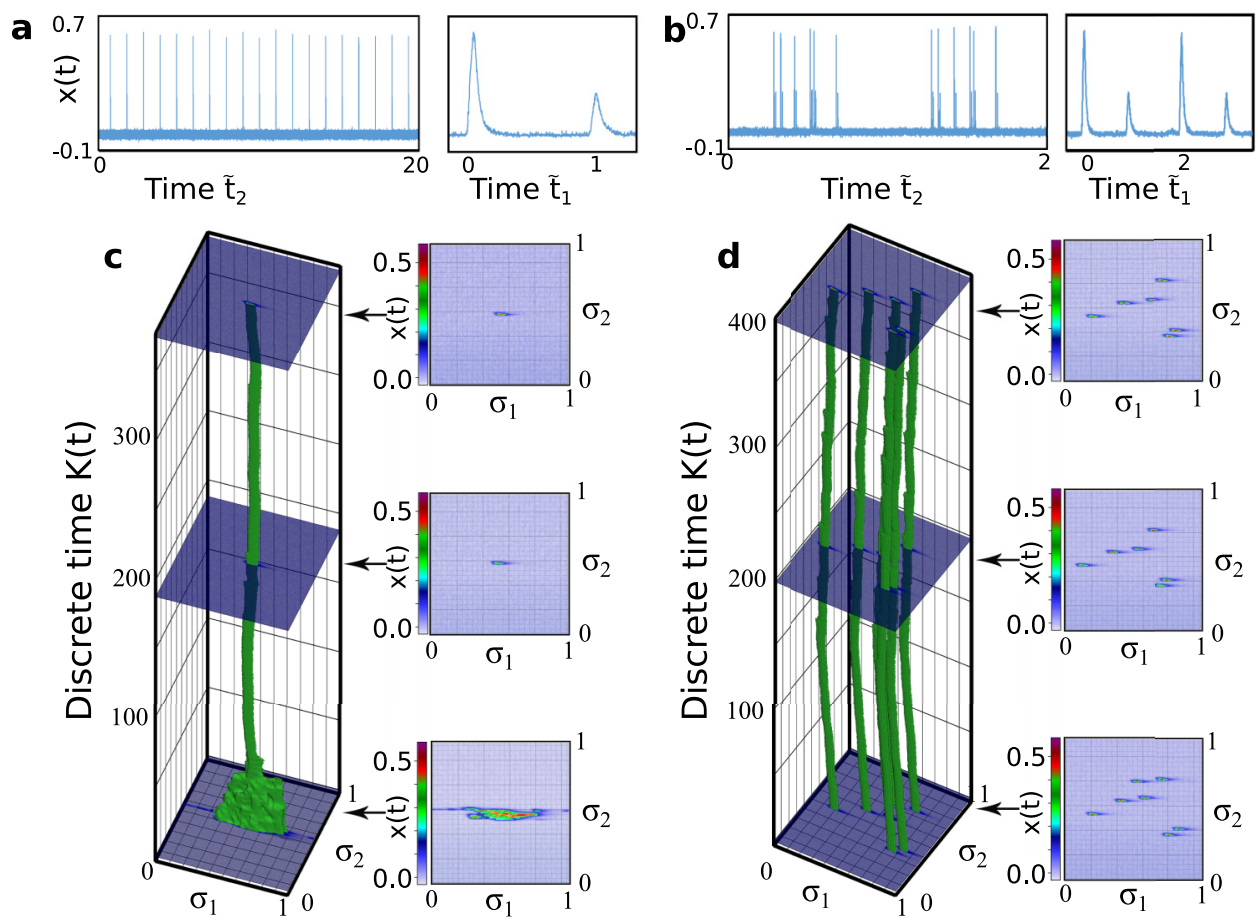

FIG. 3. Dissipative solitons obtained experimentally. Panel (a) shows the transition of chimera-like initial condition to a single DS, while in panel (b) we show multiple DSs. Asymptotic temporal waveforms of the solitons at the long delay time intervals and a zoom at the short delay intervals are shown on the top of both panels. 3D space-time plots and respective snapshots in 2D of the DS are shown below. As illustrated by the multiple DS states of panel (b), DSs are randomly located within the $2 \mathrm{D}$ space as they are induced by random initial conditions. Parameters were $\beta=1.4, m=33.5, \gamma=0.75$, and $\Phi_{0}=-0.6$.

system gave rise to different DS structures. Most DS observed structures stably persisted over long time scales.

\section{THEORETICAL ANALYSIS AND DISCUSSION}

For numerical simulations, we use a double-delayed Ikeda equation including the integral term $\delta \cdot \int_{t_{0}}^{t} x(\xi) d \xi$. Physically, this integral term corresponds to adding a highpass filter which is essential for stable two-dimensional patterns. ${ }^{20}$ The resulting equation is

$$
\begin{aligned}
\varepsilon & \frac{d x}{d s}(s)+x(s)+\delta \cdot \int_{s_{0}}^{s} x(\xi) d \xi \\
& =(1-\gamma) f[x(s-1)]+\gamma f[x(s-100)] .
\end{aligned}
$$

Here, $x(s)$ is the dynamical variable, $f(x)$ is the nonlinear transformation given by Eq. (1), $s=t / \tau_{1}$ is the normalized time, $\varepsilon=\tau / \tau_{1}$ and $\delta=\tau_{1} / \Theta$ are small time-scale parameters, with the time delay ratio set to $\tau_{2} / \tau_{1}=100 .{ }^{24}$ Relative amplitude scaling between the two feedback loops is provided by $\gamma$. The numerical model is publicly available. ${ }^{29}$ We find an excellent agreement between our experiment and numerical simulations based on Eq. (7) at parameters comparable to the experiment. In the model, multiple yet strongly damped echoes appear on a $\tau_{1}$-scale. Due to the inherent noise, only three of such echoes can be recognized in the experiment. Here, we would like to point out that in the case of DS we analyze numerical simulations based on a narrower Airy function with $m=50$. Under these conditions, DSs are stable in a significantly larger region than for $m=33.5$ as used in the experiment. This allows for a more substantiated analysis of the underlying phenomena, which is our objective. The resulting insight is transferable to the experimental system.

In Fig. 4(a), we show the bifurcation diagram scanning $\Phi_{0}$ at $m=50, \beta=1.6, \varepsilon=0.01$, and $\delta=0.009$. One can clearly identify the regions where DS and chimera dynamics can be found. The DS region $\left(\Phi_{1} \leq \Phi_{0} \leq \Phi_{2}\right)$ is located left to the discussed transcritical bifurcation, where the stable fixed point $x^{s}$ is replaced by $x^{0}\left(\Phi_{0}<-0.595\right)$. Chimeras are only supported by the system when its steady state corresponds to the fixed point $x^{s}\left(\Phi_{0}>-0.595\right)$. The DS, however, requires that after the transcritical bifurcation some of the system's solutions stabilize. In region $\Phi_{1} \leq \Phi_{0} \leq \Phi_{2}$, we therefore conclude that stable limit cycles arise in saddle-node bifurcations.

We then analyze the stability of a single DS in detail using the DDE-BIFTOOL package. ${ }^{30}$ In Fig. 4(b), the modulus of the five largest Floquet multipliers is shown for different values of $\Phi_{0}$. The largest multiplier is real and always equal to 1 , corresponding to the motion along the limit cycle. All other multipliers lie inside the unit circle, guaranteeing stability of the limit cycle. At the ending points $\Phi_{1}$ and $\Phi_{2}$ of the DS interval, the second largest and exclusively real-valued Floquet-multiplier approaches 1 following a standard square root dependency. Solitons therefore disappear at these boundaries due to a fold limit cycle bifurcation: the stable limit cycle is approached by an unstable one; they coalesce and annihilate each other. We therefore conclude that in our system the single DS structure arises via a fold limit cycle bifurcation. Details of fold limit cycle bifurcations can be found in Refs. 31 (Chap. 5.3) and 32 (Chap. 6.3). 

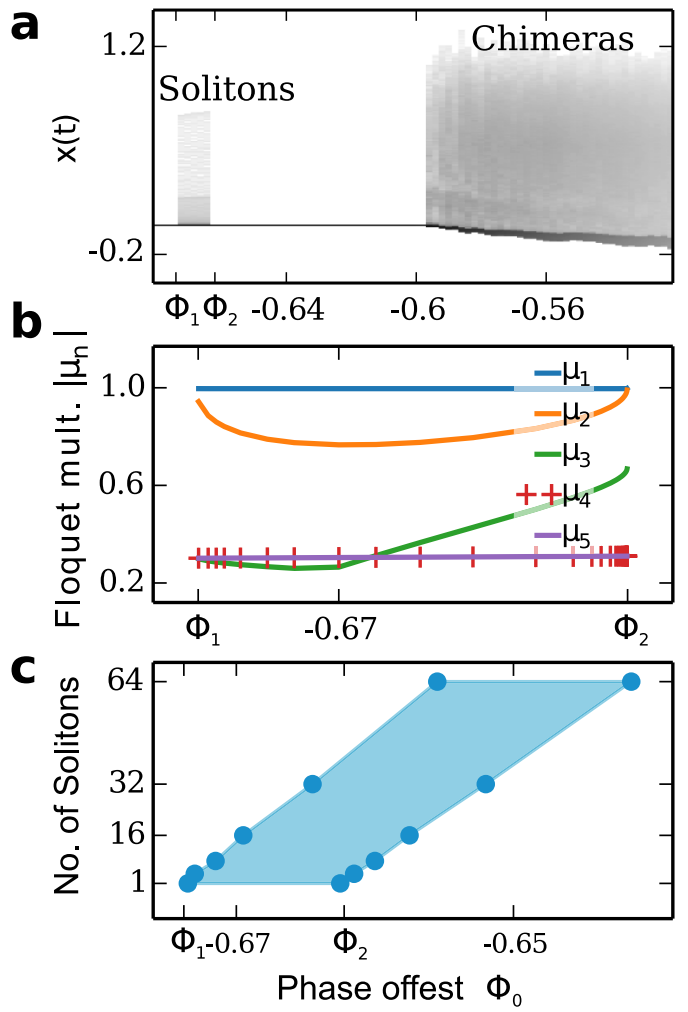

FIG. 4. Numerical investigation. Panel (a) shows the good agreement between the experiment and our numerical model for comparable parameters. Panel (b) shows the five largest Floquet-multipliers for the single DS state and their dependency on $\Phi_{0}$. The bifurcation diagram of the DS is shown in panel (c). Increasing $\Phi_{0}$ enables the system to support more and more DSs. Beyond a certain feedback strength, solutions containing few DSs are damped. Parameters fixed in all numerical simulations: $\beta=1.6, \gamma=0.83$, $m=50, \varepsilon=10^{-2}, \delta=9 \cdot 10^{-3}, \tau_{2}=100 \tau_{1}$

Figure 4(c) shows the DS's stability intervals containing up to 64 DSs obtained via direct numerical simulations. The existence window for a single DS-solution excellently agrees with the Floquet-multipliers shown in Fig. 4(b). However, for a range in $\Phi_{0}$, numerous solutions with different numbers of DSs are supported by the system. The number of DS-solutions therefore can be controlled via bias-offset $\Phi_{0}$, which agrees well with limit-cycles originating from saddle-node bifurcations as mechanism. The width in $\Phi_{0}$ of this region remains almost constant. The different numbers of co-existing DSstates for one $\Phi_{0}$ correspond to a large multi-stability, and our simulations confirm that comparable fold limit cycle bifurcation scenarios occur for these multi-DS structures. ${ }^{31}$ Such peculiar multi-stability resembles behavior in the neighborhood of a saddle-focus homoclinic orbit with one unstable direction along $x\left(t-\tau_{2}\right)$ and a focus-spiraling behavior close to the $\left[x(t), x\left(t-\tau_{1}\right)\right]$-plane. We have observed such a structure in our numerical simulations as well as in the experiment for as long as the finite signal to noise ratio permits. Cycle multi-stability is a well known behavior in the neighborhood of saddle-focus homoclinic trajectories. ${ }^{33}$ Further analysis of this complex issue is, however, beyond the scope of this work.

In our simulations, multiple DSs were seeded using initial conditions based on the experimental data. Our simulations however show that the underlying fold limit cycle bifurcation scenario can be extended to arbitrary DS location in the virtual 2D pseudo-space. We are therefore confident that more efficient spatial distributions exist and one could significantly increase the number of DS solutions, making such systems interesting for optical memory. ${ }^{13}$ Finally, we would like to stress that in addition to the DS we have also confirmed the agreement between our developed numerical model and data recorded from the experiment with regard to the chimera states. When transformed into the space-time illustrations, the agreement between simulations and experiment data is excellent.

\section{CONCLUSION}

In conclusion, we have demonstrated long living complex structures corresponding to chimeras and dissipative solitons in a highly asymmetric double delay system. After a transformation into a 2D pseudo-space, these dynamical states manifest themselves as free standing columns along the third dimension. It is the first time that this space-time analogy was shown in experiments on double delay systems. We therefore report a broad range of different dynamics, which we accompany with extensive numerical analysis. We provide a general explanation of the mechanisms causing the structuring of the system's dynamics based on the attractors of the multiple fixed-point solutions. The size of these attractors which the system's covers by its dynamics imposes global forcing to the dynamical solutions via the low-pass filter's temporal integrating effect. We anticipate that dual-delay systems and their related dynamical phenomena will represent a simple yet powerful tool for further investigations of complex self-organized motions in two dimensions.

The perfect symmetry of networks implemented in double delay systems is a fundamental asset and presents a unique opportunity to compare two dimensional models and hardware systems. Beyond the fundamental interest for the specific behavior exhibited by double delay systems, the capacity to generate and store complex high-dimensional yet stable patterns might also open new possibilities to the currently very active explorations into neuromorphic computing using nonlinear systems. In this strongly emerging topic, during a learning phase, complex patterns need to be generated and then stored, e.g. for efficient and fast classification performed by a convolution operation at the read-out layer of a Reservoir Computer. ${ }^{11,34,35}$

\section{ACKNOWLEDGMENTS}

The authors acknowledge the support of the Region Bourgogne Franche-Comté. This work was supported by Labex ACTION program (Contract No. ANR-11-LABEX-0001-0), by the EUR EIPHI program (Contract No. ANR-17-EURE0002), the BiPhoProc project (Contract No. ANR-14-OHRI0002-02), and by Deutsche Forschungsgemeinschaft (DFG) in the framework of SFB 910. Last but not least, the authors thank Jan Sieber for fruitful discussions and his valuable assistance with the DDE-BIFTOOL package.

${ }^{1}$ S. Yanchuk and G. Giacomelli, J. Phys. A: Math. Theor. 50, 103001 (2017).

${ }^{2}$ M. C. Soriano, J. Garcia-Ojalvo, C. R. Mirasso, and I. Fischer, Rev. Mod. Phys. 85, 421-470 (2013). 
${ }^{3}$ Y. Kuramoto and D. Battogtokh, Nonlinear Phenom. Complex Syst. 5, 380-385 (2002).

${ }^{4}$ D. M Abrams and S. H. Strogatz, Phys. Rev. Lett. 93, 174102 (2004).

${ }^{5}$ M. R. Tinsley, S. Nkomo, and K. Showalter, Nat. Phys. 8, 662-665 (2012).

${ }^{6}$ J. F. Totz, J. Rode, M. R. Tinsley, K. Showalter, and H. Engel, Nat. Phys. 14, 282-285 (2018).

${ }^{7}$ M. J. Panaggio and D. M. Abrams, Nonlinearity 28, R67 (2015).

${ }^{8}$ E. Schöll, Eur. Phys. J. Spec. Top. 225, 891 (2016).

${ }^{9}$ K. Saleh and Y. K. Chembo, Opt. Express 24, 25043 (2016).

${ }^{10}$ A. Argyris, D. Syvridis, L. Larger, V. Annovazzi-Lodi, P. Colet, I. Fischer, J. Garcia-Ojalvo, C. R. Mirasso, L. Pesquera, and K. A. Shore, Nature 437, 343-346 (2005).

${ }^{11}$ L. Larger, M. C. Soriano, D. Brunner, L. Appeltant, J. M. Gutierrez, L. Pesquera, C. Mirasso, and I. Fischer, Opt. Express 20, 3241-3249 (2012).

${ }^{12}$ G. Van der Sande, D. Brunner, and M. C. Soriano, Nanophotonics 6, 561-576 (2017).

${ }^{13}$ B. Romeira, R. Avó, J. M. L. Figueiredo, S. Barland, and J. Javaloyes, Sci. Rep. 6, 19510 (2016)

${ }^{14}$ A. M. Hagerstrom, T. E. Murphy, R. Roy, P. Hövel, I. Omelchenko, and E. Schöll, Nat. Phys. 8, 658-661 (2012).

${ }^{15}$ F. T. Arecchi, S. Boccaletti, S. Ducci, E. Pampaloni, P. L. Ramazza, and S. Residori, J. Nonlinear Opt. Phys. Mater. 9, 183-204 (2000).

${ }^{16}$ N. Verschueren, U. Bortolozzo, M. G. Clerc, and S. Residori, Phys. Rev. Lett. 110, 104101 (2013).

${ }^{17}$ S. Barland, J. R. Tredicce, M. Brambilla, L. A. Lugiato, S. Balle, M. Giudici, T. Maggipinto, L. Spinelli, G. Tissoni, T. Knodlk, M. Millerk, and R. Jaeger, Nature 419, 699-702 (2002).

${ }^{18}$ T. Ackemann, W. J. Firth, and G. Oppo, in Advances in Atomic Molecular, and Optical Physics, edited by E. Arimondo, P. R. Berman, and C. C. Lin (Academic Press, 2009), Vol. 57, pp. 323-421.
${ }^{19}$ O. Descalzi, M. Clerc, S. Residori, and G. Assanto, Localized States in Physics: Solitons and Patterns (Springer, 2011).

${ }^{20}$ L. Larger, B. Penkovsky, and Y. Maistrenko, Phys. Rev. Lett. 111, 054103 (2013).

${ }^{21}$ M. Marconi, J. Javaloyes, S. Barland, S. Balle, and M. Giudici, Nat. Phot. 6, 450-455 (2015).

${ }^{22}$ F. T. Arecchi, G. Giacomelli, A. Lapucci, and R. Meucci, Phys. Rev. A 45, R4225 (1992)

${ }^{23}$ G. Giacomelli and A. Politi, Phys. Rev. Lett. 76, 2686-2689 (1996).

${ }^{24}$ L. Larger, B. Penkovsky, and Y. Maistrenko, Nat. Commun. 6, 7752 (2015).

${ }^{25}$ P. Coullet, C. Elphick, and D. Repaux, Phys. Rev. Lett. 58, 431 (1987).

${ }^{26}$ W. J. Firth, Phys. Rev. Lett. 61, 329 (1988).

${ }^{27}$ G. Giacomelli, F. Marino, M. A. Zaks, and S. Yanchuk, Europhys. Lett. 99, 58005 (2012).

${ }^{28}$ S. Yanchuk and G. Giacomelli, Phys. Rev. Lett. 112, 174103 (2014).

${ }^{29}$ B. Penkovsky, D. Brunner, and L. Larger, See https://bit.ly/2Q3UCdz for the numerical code employed in the system's numerical simulation.

${ }^{30}$ K. Engelborghs, T. Luzyanina, and D. Roose, "Numerical bifurcation analysis of delay differential equations using DDE-BIFTOOL," ACM Trans. Math. Softw. 28(1), 1-21 (2002).

${ }^{31}$ Y. Kuznetsov, Elements of Applied Bifurcation Theory (Springer, 1995).

${ }^{32}$ E. Izhikevich, Dynamical Systems in Neuroscience: The Geometry of Excitability and Bursting (The MIT Press, 2007).

${ }^{33}$ L. P. Shilnikov, A. L. Shilnikov, D. V. Turaev, and L. Chua, Methods of Qualitative Theory in Nonlinear Dynamics (World Scientific, 2001).

${ }^{34}$ D. Brunner, M. C. Soriano, C. R. Mirasso, and I. Fischer, Nat. Commun. 4, 1364 (2013).

${ }^{35}$ Q. Vinckier, F. Duport, A. Smerieri, K. Vandoorne, P. Bienstmann, M. Haeltermann, and S. Massar, Optica 2, 438-446 (2015). 